Service social

\title{
Vers un développement international du travail social de groupe. L'établissement des échanges frontaliers culturels et linguistiques.
}

\section{Paul Taylor}

Volume 46, numéro 2-3, 1997

Groupes - Symposium 1997

URI : https://id.erudit.org/iderudit/706756ar

DOI : https://doi.org/10.7202/706756ar

Aller au sommaire du numéro

Éditeur(s)

École de service social de l'Université Laval

ISSN

1708-1734 (numérique)

Découvrir la revue

Citer cet article

Taylor, P. (1997). Vers un développement international du travail social de groupe. L'établissement des échanges frontaliers culturels et linguistiques. Service social, 46(2-3), 11-31. https://doi.org/10.7202/706756ar

\section{Résumé de l'article}

Comment pouvons-nous créer une praxis éducative du travail social de groupe, à un niveau international, engagée dans une lutte collective pour une société radicalement plus juste, tout en respectant pleinement la notion de différence et d'identité culturelle inhérente à tout groupe? Comment définissons-nous les situations limites conflictuelles (tolérance, langue, culture, ignorance) dans lesquelles nous travaillons? Ce texte permet d'explorer la complexité de ces questions et d'identifier quelques-uns des échanges frontaliers qui se développent entre l'international et l'interculturel. 


\section{Vers un développement international du travail social de groupe L'établissement des échanges frontaliers culturels et linguistiques}

Paul TAYLOR

Comment pouvons-nous créer une praxis éducative du travail social de groupe, à un niveau international, engagée dans une lutte collective pour une société radicalement plus juste, tout en respectant pleinement la notion de différence et d'identité culturelle inhérente à tout groupe? Comment définissons-nous les situations limites conflictuelles (tolérance, langue, culture, ignorance) dans lesquelles nous travaillons? Ce texte permet d'explorer la complexité de ces questions et d'identifier quelques-uns des échanges frontaliers qui se développent entre l'inter-national et l'inter-culturel.

How can we create an educative parxis of social groupwork, at an international level, committed to the collective, human struggle for a radically more just society, while fully respecting the notion of difference and cultural identity in any given group? How do we define the socially and historically constructed boundaries of our own practice? How do we analyze the conflicting limit situations (tolerance, language, culture, ignorance) within which we work? This paper seeks to explore the complexity of these questions and to identify some of the developmental "border crossings" between the inter-national and the inter-cultural. 


\section{INTRODUCTION}

Le titre, déjà, fait problème. Où le mot « international " devrait-il se trouver? Est-il question du développement international du travail de groupe ou du développement d'un travail de groupe international? Dans le dernier cas, nous devrions probablement parler plutôt d'un travail de groupe transculturel. Dans le premier cas, il ne s'agit pas vraiment du travail de groupe, mais du contexte sociopolitique dans lequel celui-ci prendrait place. Dans les deux cas, qui fait le. travail (de groupe) et qui est censé faire le développement?

Formuler la question de la sorte n'est pas seulement une manière facile de trouver une introduction. En fait, si nous arrivons à être plus clairs sur cette question et surtout si nous avons un début de réponse à la fin de ce colloque international, nous saurons que nous avons avancé vers de nouvelles dimensions du travail de groupe.

Deux raisons principales font que le simple fait de définir la question est difficile. La première est que presque chaque aspect de notre vie quotidienne, personnelle et professionnelle, est de plus en plus soumis aux forces de la mondialisation ou globalisation. Par ailleurs, nous commençons à comprendre comment le Web, inter-, extra-, supra- (pour employer les images les plus fortes des nouvelles technologies), réduit notre “ espace vital » mental. Les gens se trouvent dorénavant à une distance de courriel; l'information et la communication sont désormais possibles 24 heures par jour à la grandeur de la planète. Qu'on le veuille ou non, qu'on le sache ou non, nous sommes maintenant tous pris dans la toile de l'Internet : nous sommes devenus des surfeurs intergalactiques, des correspondants intercontinentaux et des acheteurs internationaux.

Loin d'être un écoumène humanisant ou idéalisant ${ }^{1}$, ce nouvel ordre social est une véritable méga-économie. Le processus de la mondialisation est essentiellement économique, dirigé par les multinationales et les acteurs du marché des valeurs global, alimenté par la croyance inébranlable que le libre-échange signifie le droit de faire des profits partout, en tout temps et au détriment de n'importe qui. Les vieilles cartes politiques multicolores de l'Europe, de l'Orient

1. A. Berque, Êtres humains sur la terre: Principes d'éthique de l'écoumène, Paris, Gallimard, 1996. 
et des Amériques ont fait place à de nouvelles cartes d'unions et d'alliances supranationales d'échange, désignées comme des « zones " pour l'usage exclusif du dollar, de l'ECU ou du yen.

Cette homogénéisation de la culture, qui va de la standardisation même architecturale de presque toutes nos grandes villes jusqu'à l'omniprésent McDo, en passant par l'uniforme jeans et l'hégémonie linguistique de l'anglais, est un phénomène surdéterminé ${ }^{2}$ par l'économique. Pour bien saisir plusieurs de ces aspects, nous devrions commencer par comprendre les lois fondamentales de l'économie moderne.

II peut paraître difficile d'établir un lien direct avec le travail de groupe, mais la raison pour laquelle notre question de départ pose problème, c'est qu'il existe un contre-courant énorme - et parfois terrifiant - en opposition à cette universalisation obligée. II semble presque forcé que plus nous globalisons, plus nous localisons. Dans presque tous les pays du monde, on assiste à une renaissance du nationalisme et du régionalisme : revendications des peuples autochtones d'Amérique du Nord et du Sud; le Timor oriental, le Pays basque, la Corse, la Macédoine, les Kurdes..., propositions de dissolution du Parlement en Écosse et au pays de Galles, troubles incessants en Irlande, demandes pour une Palestine libre, la Bosnie, la Malaisie... et le Québec. La liste est longue et souvent marquée par les conflits, le terrorisme et la contre-oppression.

Ce sens de l'identité nationale est complexe, car il correspond à une communauté d'intérêts fondés sur une histoire partagée, sur des valeurs culturelles spécifiques, sur des allégeances religieuses, sur une pureté ethnique, sur des revendications territoriales pour la terre ou la mer ou encore sur des zones linguistiques bien identifiées. Les gens veulent ré-affirmer leur individualité, leur identité, leur droit à ne pas être perdus individuellement ou collectivement, dans un macro-monde anonyme, dépersonnalisé où leur seul bien est économique.

D'une certaine façon, tout cela est parfaitement compréhensible, mais nous devons nous demander comment nous le comprenons. Il est important de réaliser que jusqu'ici ce mouvement vers le

2. Surl'idée de « surdétermination », (overdetermination, Uberdeterminierung), voir L. Althusser, For Marx, Londres, Allen Lane, 1969. 
nationalisme demeure largement sous-documenté. Les historiens ont décrit la grandeur et la décadence du nazisme, mais nous ne comprenons encore généralement pas les relations qui existaient entre Hitler, le IIle Reich et le peuple allemand. Les politiciens ont déploré le scandale de la purification ethnique en Bosnie et au Rwanda et l'aide humanitaire s'est précipitée pour arrêter le flot de souffrance humaine, mais nous n'en comprenons toujours pas les causes sous-jacentes. Les anthropologues et les sociologues ont relevé les facteurs inhérents à l'opposition de différents « nationalismes ". Mais quelles sont les différentes théories qui étayent ces énoncés des faits? Sans une base théorique solide, le danger est toujours présent que nous trouvions une bonne réponse, mais à la mauvaise question.

Les praticiens du travail de groupe devraient commencer à explorer ensemble un programme de recherche-action, à l'intérieur de leurs pratiques respectives, qui se pencherait sur ce problème fondamental.

Nous nous éloignons apparemment de la pratique quotidienne du travail de groupe en nous perdant dans quelque théorisation académique. Permettez-moi donc d'illustrer brièvement ce besoin de recherche collective.

Une des questions clés à se poser porte sur l'État. Nous ne pouvons commencer à élaborer l'idée de travail de groupe international sans nous demander ce que nous entendons par inter-nation. Quelle est la relation entre l'État et la Nation? Plusieurs des différents mouvements nationalistes semblent déterminés à réinventer l'idée de l'État-nation qui a donné une multiplicité de royaumes et de principautés à travers l'Europe au cours des $15^{\circ}$ et $16^{e}$ siècles. La Ligue du Nord en Italie en est aujourd'hui un exemple typique. Ou le débat en France au sujet des contrôles de l'immigration, qui définit en fait la citoyenneté exclusivement en fonction des principes de la Révolution et du rapport privilégié entre le citoyen et la République française. Ou, encore, le débat plus large en Europe où l'on réclame d'abord une monnaie commune; ensuite, une monnaie unique dépend de deux visions différentes de l'Europe. L'une a la forme multiple d'une fédération d'États-nations, alors que l'autre a la forme simple d'une union supranationale. 
Nous pouvons très bien vouloir réinventer l'État-nation, mais, en même temps, nous ne pouvons désinventer l'idée plus moderne de l'État-providence. Tous les pays semblent maintenant partager l'idée que, suivant un principe général de "subsidiarité sociale ", l'État devrait assumer certaines responsabilités qui excèdent les moyens ou les compétences de ces citoyens pris individuellement, par exemple en matière de sécurité sociale, d'assistance sociale, d'assurance maladie, de régimes de retraite, de protection pour les travailleurs comme pour les jeunes, les personnes âgées ou handicapées. Cependant, comme nous le savons en tant que travailleurs dans nos différents champs de pratique, l'essence même de l'assistance sociale est qu'elle devrait se faire localement, au moins en ce qui a trait à la fourniture de l'aide. Le travail social lui-même peut être défini comme l'intermédiaire institutionnalisé entre l'État et les besoins de la personne.

Autre élément du problème. Étant donné la taille du budget de la sécurité sociale, les besoins grandissants pour une aide à long terme, la non-contribution au système à cause du chômage, une population de plus en plus âgée et le besoin de soutenir les régimes de rentes en jonglant avec des portefeuilles d'investissements, etc., le financement de l'aide sociale est devenu, comme l'industrie de l'armement, une autre industrie internationale. La politique de l'aide sociale est mise en œuvre localement, mais définie globalement.

Ainsi, si nous allions demander, dans les différents pays, " Qui travaille en soins communautaires? ou qui travaille en éducation communautaire? ", la réponse devrait traverser le filtre de notre propre compréhension de l'État, de la nation et de la communauté. Par exemple, la notion de soins à la communauté aux États-Unis, en France, en Grèce ou dans les pays scandinaves ne renvoie pas aux mêmes pratiques ni aux mêmes principes d'action sociale ni à la même politique sociale. Une des barrières, invisibles mais bien réelles, au travail de groupe transnational en soins communautaires, par exemple, est que les intervenants peuvent très bien s'adresser à la même clientèle, utiliser le même jargon professionnel, ils n'ont quand même pas la même compréhension fondamentale de ce qu'est le "soin à la communauté ". 


\section{INTER-NATIONALISER LE TRAVAIL DE GROUPE}

Par où faut-il donc commencer? Par chance, on connaît certains des ingrédients. Ainsi, le développement (international) du travail de groupe (international) nécessite :

a) une compréhension de "l'identité nationale ";

b) une compréhension de "l'appartenance culturelle »; et par conséquent

c) une compréhension de la façon dont l'identité nationale et l'appartenance culturelle influent sur la dynamique de tout groupe.

Ma première expérience d'échanges frontaliers en matière de travail de groupe s'est déroulée il y a plusieurs années, dans la partie ouest de l'Afrique. Je m'y suis particulièrement intéressé à la gestion des crises et à la résolution des conflits entre les différentes cultures du Sahel alors que je travaillais pour la Croix-Rouge internationale. Ce creuset de bonnes intentions était révélateur : la crise était locale, due à la sécheresse, mais le conflit était plus souvent qu'autrement européen.

Plus récemment, alors que j'enseignais et travaillais en Europe, je me suis penché sur un aspect particulier de la vie en groupe, la réunion. Le rôle et la fonction d'une réunion ne sont pas les mêmes dans les différentes cultures d'Europe : pour certains, la réunion sert à transmettre de l'information; pour d'autres, elle est le lieu où l'on confirme des décisions déjà prises ailleurs. Cependant, chaque réunion possède un code et une procédure qui lui sont propres, un ordre hiérarchique et des exceptions, et il est parfois très difficile d'apprendre comment s'y comporter et quelle contribution y apporter (ou pas) ${ }^{3}$.

L'étude de Bollinger et Hofstede constitue un point de départ valable ${ }^{4}$. Au moyen d'entrevues très poussées, ils ont essentiellement cherché à dresser une typologie du comportement managérial, une classification que j'utilise volontiers pour la raison qui suit. Mon

3. Pour une étude plus détaillée, voir P. Taylor, «The Linguistic and Cultural Barriers to Cross-National Groupwork », Groupwork, vol. 7, n 1, 1994, p. 7-22.

4. D. Bollinger et $\mathrm{G}$. Hofstede, Les différences culturelles dans le management, Paris, Éditions d'Organisation, 1987. 
hypothèse de travail est que les groupes sociaux ont tendance à se conformer aux modèles dominants de management en usage dans n'importe quel pays donné. Ces typologies de départ de Bollinger et Hofstede ne sont pas des stéréotypes qui viendraient simplement conforter nos différents préjugés nationaux : il s'agit de comportements typiques ou standardisés, ainsi que le font ressortir les interviewés eux-mêmes.

Bollinger et Hofstede ont étudié 53 pays et bâti une échelle différentielle qui permet de comparer entre eux divers modèles nationaux. Chaque pays y possède un rang (1-53) et un score individuel (normalement entre 0 et 100-110). Leur recherche relève quatre éléments clés au sujet desquels les perceptions diffèrent considérablement d'une frontière à l'autre.

\section{La distance hiérarchique}

Comment une société donnée perçoit-elle le niveau d'inégalité ou la distance entre ceux qui détiennent le pouvoir et ceux qui sont soumis à ce pouvoir? Comment les détenteurs du pouvoir consultent-ils? De quelle façon est-ce qu'ils délèguent, évaluent une opinion contraire, prennent des initiatives, personnalisent les responsabilités?

Par le recours à cette méthode pour mesurer le continuum du pouvoir, en établissant la dynamique psychologique et sociale qui existe dans tout groupe, nous pouvons commencer à calculer la distance hiérarchique relative (DHR). Ainsi, les pays qui ont une DHR élevée préféreront un mode de communication et d'interaction qui tend à être autocratique et paternaliste. Une DHR basse dénote une préférence pour les processus consultatifs et démocratiques. Les pays latins ont tendance à avoir une DHR plus élevée que les pays germaniques; les cultures de haute technologie ont une DHR faible. Enfin, les pays pauvres, où la distinction entre les puissants et les faibles est évidente, ont normalement une DHR élevée. 
Distance hiérarchique relative

\begin{tabular}{ccc}
\hline Rang & & Score \\
\hline 5 & Mexique & 81 \\
7 & Arabie saoudite & 80 \\
14 & Brésil & 69 \\
16 & France & 68 \\
24 & Chili & 63 \\
33 & Japon & 54 \\
38 & États-Unis & 40 \\
39 & Canada & 39 \\
44 & Royaume-Uni & 35 \\
52 & Israël & 13 \\
\hline
\end{tabular}

Note: Un haut score correspond ici à une distance hiérarchique élevée et un bas score à une distance hiérarchique basse.

\section{L'individualité}

Des sociétés différentes bâtissent le continuum « société-communauté-famille-individu » de manières différentes, certaines en sociétés beaucoup plus communales, d'autres beaucoup plus centrées sur la personne. Les premières mettent l'accent sur le nous, sur l'égalité sociale, sur le rôle des individus plutôt que sur leur personnalité. Les dernières privilégient le je, la différence sociale et la distinction entre les individus. II va de soi que les processus d'immigration et d'émigration à l'intérieur et à l'extérieur de ces groupes diffèrent.

Certaines cultures sont-elles plus axées sur la coopération ou sur la compétition? Le changement social suit-il un plan évolutif, logique et ordonné sans révolution majeure? Ou oscille-t-il de gauche à droite, créant et changeant les alliances, rejetant les inutiles ou les incompétents? 
Niveau d'individualisation

\begin{tabular}{ccc}
\hline Rang & & Score \\
\hline 1 & États-Unis & 91 \\
3 & Royaume-Uni & 89 \\
4 & Canada & 80 \\
11 & France & 71 \\
19 & Israël & 54 \\
22 & Japon & 46 \\
26 & Brésil & 38 \\
27 & Arabie saoudite & 38 \\
32 & Mexique & 30 \\
38 & Chili & 23 \\
\hline
\end{tabular}

Note : Un haut score correspond à une société individualiste et un bas score, à une société d'orientation communautaire.

\section{Le genre}

Les rôles et les attentes fondés sur le genre sont sans contredit une composante essentielle du travail de groupe, mais ils n'en sont pas pour autant universels. Dans certains pays, les rôles liés à un statut élevé sont masculins d'office et c'est l'homme qui est habituellement avantagé sur les plans social, culturel et économique. Bollinger et Hofstede ont distingué de façon rapide entre, d'une part, une " culture mâle » qui établit une nette distinction entre les rôles masculins et féminins et, d'autre part, une « culture féminine » à l'inté-

Les conclusions sont très éloquentes. Les cultures mâles tendent à être compétitives, centrées sur le statut, orientées vers le produit, tout en étant dépendantes de la technologie. Dans les cultures féminines, particulièrement dans les petits groupes, on est plutôt orienté sur la méthode, on s'intéresse davantage à la coopération et à l'échange, on est centré sur la compétence plus que sur le statut et, enfin, on met plus à profit les ressources humaines que les ressources technologiques. 
Différenciation des rôles selon le genre

\begin{tabular}{ccc}
\hline Rang & & Score \\
\hline 1 & Japon & 95 \\
6 & Mexique & 69 \\
9 & Royaume-Uni & 66 \\
15 & États-Unis & 62 \\
23 & Arabie saoudite & 53 \\
24 & Canada & 52 \\
27 & Brésil & 49 \\
29 & Israël & 47 \\
36 & France & 43 \\
46 & Chili & 28 \\
\hline
\end{tabular}

Note: Un haut score correspond ici à une culture masculine et un bas score, à une culture féminine.

\section{La prise de risques}

Comment voyons-nous l'avenir? Comment vivons-nous le changement? Certains groupes ont besoin d'une réglementation claire pour dissiper toute équivoque, et ils doivent s'appuyer sur une procédure précise, où l'appartenance ou non au groupe est bien établie et où l'on peut toujours en référer à une instance supérieure. D'autres groupes s'épanouissent à l'enseigne de l'initiative, de l'intuition, de la liberté de changer et de s'adapter à leur gré, parce qu'ils sont capables de prendre plus de risques personnels et de gérer une plus grande responsabilité individuelle.

Certains pays, comme les États-Unis, sont faciles à identifier comme étant des « cultures de risque ", alors que d'autres comportent un haut niveau de contrôle et accordent un statut élevé à l'" expert " et au consultant, au « docteur » qui peut tout arranger. 
Prise de risques

\begin{tabular}{ccc}
\hline Rang & & Score \\
\hline 7 & Japon & 92 \\
10 & France & 86 \\
11 & Chili & 86 \\
18 & Mexique & 82 \\
19 & Israël & 81 \\
22 & Brésil & 76 \\
27 & Arabie saoudite & 68 \\
42 & Canada & 48 \\
43 & États-Unis & 46 \\
47 & Royaume-Uni & 35 \\
\hline
\end{tabular}

Note: Un haut score signifie que la prise de risques est menaçante et doit être contrôlée, alors qu'un bas score veut dire que la prise de risque est une source de libération à exploiter.

Une vue d'ensemble de ces quatre dimensions culturelles révèle, bien que ce ne soit que d'une façon numérique, les points de ressemblance et de différence entre les diverses cultures-nations.

\begin{tabular}{lcccc}
\hline & $\begin{array}{c}\text { Distance } \\
\text { hiérarchique }\end{array}$ & Individualité & Genre & $\begin{array}{c}\text { Prise de } \\
\text { risques }\end{array}$ \\
\hline Brésil & 14 & 26 & 27 & 22 \\
Canada & 39 & 4 & 24 & 42 \\
Chili & 24 & 38 & 46 & 11 \\
France & 16 & 11 & 36 & 10 \\
Israël & 52 & 19 & 29 & 19 \\
Japon & 33 & 22 & 1 & 7 \\
Mexique & 5 & 32 & 6 & 18 \\
Arabie saoudite & 7 & 27 & 23 & 27 \\
Royaume-Uni & 44 & 3 & 9 & 47 \\
États-Unis & 38 & 1 & 15 & 43 \\
\hline
\end{tabular}




\section{LE TRAVAIL SOCIAL DE GROUPE ET LES FRONTIÈRES}

Le travail de groupe international n'est pas un énorme caméléon qui se fonde aux couleurs d'un étendard national ou qui s'adapte mentalement à la culture dominante où qu'elle soit. Si c'était le cas, nous ne pourrions jamais en déterminer l'objectif fondamental. Le travail de groupe est plutôt une réunion d'idéologies, de pratiques et de processus de communication et d'interaction sociale reliés à la société qui les produit et qu'ils reproduisent en retour. Le travail de groupe transfrontalier correspond à un changement dans la mesure et donc dans la complexité, mais pas dans les fondements.

Ce qu'il faut, ce n'est pas simplement décrire les différences nationales, mais élaborer un cadre d'analyse qui nous permettra d'aller plus loin. Bien qu'il soit toujours intéressant d'approfondir un comportement en situation interculturelle, notre tâche première consiste à découvrir où cette situation pourrait avoir lieu. En d'autres mots, qu'advient-il du concept de " travail de groupe " quand nous y ajoutons l'adjectif « international »? L'objectif d'une création d'alliances visé par ce symposium même constitue en fait une réponse à la question où? plutôt qu'à la question comment? Quelques exemples nous permettent ici d'illustrer nos propos.

La nécessité d'être poli. Au début de la vie de groupe, au cours des premières rencontres, quand on piétine, qu'on apprend à se connaître, dans presque toutes les cultures on travaille dans un cadre de politesse. Mais être simplement poli ne suffit pas. Comment entendez-vous être poli? Partant du principe qu'aucune rencontre interpersonnelle n'est "sans visage " (et l'idée de "visage " est cruciale ici), nous pouvons définir trois différents processus de savoir-vivre, chacun fonctionnant à l'intérieur d'une dynamique de distance et de pouvoir 5 .

Le premier est un processus de déférence, par lequel les membres du groupe se comportent mutuellement avec un grand respect (recourant à des formules de politesse, ou employant le vous ou

5. Pour une analyse plus détaillée, voir R. Scollon et S. Wong Scollon, Intercultural Communication, Oxford et Cambridge, Blackwell, 1995, p. 33-49. 
Usted ou une forme équivalente) et gardent leurs distances. Ce processus est courant dans plusieurs cultures orientales et sudaméricaines.

Le deuxième peut être défini comme un processus de solidarité, où s'exprime un souci d'égalité mais qui implique qu'il existe déjà une relation interpersonnelle étroite, ou tout au moins le désir d'en établir une. Ce modèle est fréquent à l'intérieur d'une culture nord-américaine professionnelle et d'affaires.

Le troisième processus reflète un sentiment de politesse hiérarchique où l'on est très conscient de l'inégalité du statut et du pouvoir.

L'important dans ces situations est que le processus de politesse soit symétrique. Ce qui est de toute évidence difficile dans une rencontre entre des cultures à distance hiérarchique élevée et, par exemple, des cultures individualistes.

Être égocentrique. J'ai fait récemment une rare visite à Londres en compagnie d'un groupe d'éducateurs français afin de participer à un séminaire interculturel. Au cours d'une des premières discussions en plénière, durant laquelle nous préparions le programme, une étudiante anglaise, qui était censée être l'" hôte " de la séance, s'exclama : "Mais je ne vois pas comment mes besoins en matière d'apprentissage pourront être comblés. Ce que je recherche, c'est... " Le groupe est resté sidéré, pratiquement incapable de répondre, sinon pour satisfaire les désirs de l'étudiante. Où donc l'apprentissage égocentrique se situe-t-il dans la construction culturelle d'un groupe, étant donné l'interrelation entre la distance hiérarchique relative et l'individualisation, particulièrement dans les débuts de la vie du groupe? L'étudiante croyait, de toute évidence, qu'elle négociait, alors que plusieurs autres personnes ont trouvé son attitude bêtement provocatrice.

Un groupe pour femmes seulement? Appartenant à une culture où il est tout à fait normal qu'un groupe de femmes veuillent travailler ensemble, j'ai souvent essayé de comprendre pourquoi les étudiants et travailleurs sociaux français ont toujours repoussé cette idée. II n'est pas étonnant que de nombreux hommes répugnent à cette perspective en principe, mais ce qui est surprenant, c'est le fait que plusieurs femmes ne voient pas non plus l'utilité d'" exclure les hommes » du groupe. 
C'est en fait l'opposé du syndrome que j'ai observé quand une collègue anglaise est venue à notre université. Je lui expliquai une partie de la dynamique sous-tendant le régime d'éducation en France. " Je peux tolérer tout cela, dit-elle, tout sauf le sexisme. " II reste que le problème n'est pas un problème de tolérance. La difficulté est d'être clair sur ce qui constitue du sexisme ou, ce qui est encore plus difficile, ce qui constitue de l'antisexisme dans les différentes cultures.

\section{LA RENCONTRE TRANSFRONTALIÈRE}

Étant donné les niveaux différents auxquels nous fonctionnons professionnellement en groupe, il apparaît important d'examiner la complexité d'une rencontre transfrontalière. Nous pouvons supposer qu'il s'agit ici essentiellement de contacts interpersonnels plutôt qu'interinstitutionnels. Des rapports transfrontaliers organisationnels ou politiques appellent une analyse différente, mais même dans le travail de groupe ces contacts sont inévitablement pluridimensionnels.

La relation entre la personne travaillant en groupe et son groupe d'une autre culture-nation peut s'établir par :

- la rencontre et l'intégration au groupe d'une personne d'une autre culture-nation;

- la rencontre et l'association avec un groupe d'une autre culturenation;

- la rencontre avec une personne ou un groupe qui représentent les institutions de leur culture-nation. Par exemple, une personne représentant le système d'éducation ou d'assistance sociale, la police, les autorités civiles ou religieuses, une équipe nationale en sport.

À linverse, la personne travaillant en groupe et son groupe peuvent être intégrés dans l'autre culture-nation. En ce sens, la culturenation qui est chez elle peut rencontrer l'autre culture-nation:

- une personne se retrouve dans un groupe " étranger ";

- le groupe qui est chez lui travaille à l'intérieur d'une culture qui lui est étrangère; 
- le groupe ou les individus qui sont chez eux représentent leur culture ou leur nation ou ses institutions.

Par exemple, certains participants étrangers à ce colloque qui sont venus ici de leur propre chef, par intérêt personnel, peuvent se voir en individus se retrouvant dans un groupe " étranger ", tout en étant traités en personnes représentant les institutions de leur culture-nation. Que va-t-il se passer dans les petites séances de groupe? Le groupe anglophone dominant va-t-il dans les faits se comporter comme rencontrant un groupe d'une autre culturenation? Comment allons-nous décider de l'ambiance et des règles de notre rencontre internationale?

Aucune rencontre internationale ou interculturelle n'est neutre. Même si nous affirmons haut et fort Vive la différence! ou souscrivons à un libéralisme qui prône l'égalité par la différence, nous ne pouvons échapper au fait que, dans la mesure où une culture-nation cherche à dominer l'autre ou cède sa place à l'autre en vertu de quelque attribut réel ou imaginaire, positif ou négatif, l'interprétation que l'on fera de la rencontre sera radicalement différente.

Nous en savons assez sur la complexité des changements dans le contenu de l'interaction à l'intérieur des petits groupes formés de dyades et de triades pour ne pas nous précipiter dans la dimension d'une véritable rencontre multi-nationale. Illustrons ceci par la rencontre à deux. La culture ou la nation $A$ peut dominer et chercher à dominer $B$, et leur rencontre prend place dans un environnement $A b$. La culture ou la nation $B$ peut dominer ou chercher à dominer $A$, d'où une rencontre passablement différente $a B$. Ou encore $A$ et $B$ pourraient se rencontrer sur la base d'un statut équivalent ou d'un pouvoir égal, $A B$.

Intéressons-nous à nouveau à une visiteuse et disons qu'elle est Allemande ou Française. Elle peut se considérer comme une praticienne expérimentée outre-frontière, et trouver plaisir à travailler dans une langue et une culture qui ne sont pas les siennes. D'autres participants au colloque, soucieux d'évaluer la caractéristique « autre » de l'expérience de la visiteuse, s'emploient à redresser l'équilibre culturel, mais la considèrent finalement comme typique du travail social et de la pratique de groupe en Allemagne ou en France. 
Le point de départ consiste à accepter le jugement perçu de l'individu ou du groupe quant à l'endroit où ils se situent dans la rencontre. C'est une autre façon de mettre l'accent sur l'importance des deux visions différentes de la culture que j'ai décrites comme étant l'emic et l'etic 6 . Les deux perspectives sont forcément culturocentriques, au sens où la culture est l'un des principaux moyens d'exprimer son identité et son appartenance sociale. Le processus est fondé sur l'intégration, l'acculturation et la socialisation, qui convergent pour fournir à l'individu, au groupe et à la communauté un centre culturel. Nous apprenons l'appartenance, nous apprenons à nous rattacher à un certain mode de vie, à certaines valeurs, à avoir des racines et à reconnaître ceux qui partagent les mêmes racines. Le culturocentrisme est au groupe ce que l'égocentrisme est à l'individu. Je dois partir du fait que je suis qui je suis avant de vous demander qui vous êtes.

La distinction entre emics et etics n'est pas nouvelle; elle reflète le développement d'idées qui prennent leur source dans la théorie de Tajfel sur le "groupe nous " et dans le débat plus large entre ceux "du dedans" et ceux "du dehors " ${ }^{7}$. La perspective emic vient de l'intérieur, de l'expérience vécue; elle se bâtit à partir des valeurs inhérentes au système. La vision etic naît de l'extérieur et de l'expérience apprise, fondant son jugement sur des valeurs extérieures au système. Je crois cependant que nous commençons à peine à voir son potentiel en tant qu'outil du travail de groupe transculturel et transnational.

\section{DE L'INTER- AU TRANS-CULTUREL}

Le travail social de groupe est souvent utilisé comme méthode d'intégration sociale, de réhabilitation individuelle ou d'assimilation culturelle. Le groupe est à la fois une façon économique de travailler et, plus particulièrement, l'un des meilleurs moyens de créer la solidarité et la cohésion nécessaires à une action sociale efficace. Étant

6. P. Taylor, 1994, p. 9.

7. Voir H. Tajfel, "An integrative theory of intergroup conflict ", dans W. Austin et S. Worchel (dir.), The Social Psychology of Intergroup Relations, California, Brooks Cole, 1979; T. Headland, K. Pike et M. Harris (dir.), Emics and Etics : the Insider/Outsider Debate, Newbury Park , Sage, 1990; H. Atmanspacher et G. Dalenoort, Insider versus Outsider, Berlin, Springer-Verlag, 1994. 
donné que les problèmes entraînés par le chômage, la discrimination, la pauvreté et la santé précaire ne tiennent aucun compte des frontières nationales, il est naturel en un certain sens que les intervenants de groupe regardent outre-frontière pour trouver des solutions à des difficultés même très locales.

Nous ne pouvons faire abstraction de cette dynamique entre le micro et le macro. Toutefois, si les intervenants situent leur réflexion à un niveau micro-local, il est possible qu'ils puissent venir en aide à un certain nombre de personnes, mais il est également possible qu'ils ne puissent aller au-delà du traitement des symptômes du problème. La distinction faite par Wright Mills est importante : il ne faut pas confondre une difficulté personnelle et le fondement d'un problème social ${ }^{8}$.

Si nous travaillons avec un groupe de sans-emploi ou avec un groupe de malades mentaux, nous aurons fort à faire pour susciter à l'intérieur du groupe la confiance et l'échange qui auront éventuellement des résultats très positifs chez chaque personne. Cependant, si nous nous attardons à ces horizons individuels, nous ne ferons rien pour combattre le problème social qu'est le chômage, ni les facteurs sociaux qui provoquent et favorisent la maladie mentale. Très rapidement, le travail social en groupe finit par " blâmer les victimes ".

On est fondé de poser la question : quelle proportion de notre temps professionnel se passe vraiment à travailler en fonction de cet horizon macro-sociopolitique? Mais même cette question présuppose qu'on possède déjà les compétences nécessaires pour travailler à un niveau national et international. À l'extrême, nous trouvons, par exemple, des intervenants qui font un travail de création avec des nouveaux immigrants, mais qui voient leur travail uniquement sous un angle monoculturel. Ils ne cherchent aucunement à tisser des liens avec le pays d'origine de ces immigrants. Pensons aussi à des groupes de retraités qui ne s'intéresseraient plus au monde du travail, un monde qui ne peut se soustraire aux influences du multinationalisme.

8. C. Wright-Mills, L'imagination sociologique, Paris, Maspero, 1983, p. 10. 
II ne faut pas déduire de mon propos que la santé, l'éducation, la pauvreté, l'emploi, la sécurité sociale, l'immigration, etc., ne devraient pas être considérés comme des réalités locales, que de tels problèmes sont vécus de la même façon dans toutes les cultures. Loin de là : il existe beaucoup trop de facteurs, économiques, moraux, environnementaux et politiques qui font en sorte que chaque expérience est vécue différemment d'un pays à l'autre. Le présent colloque nous fournit l'occasion d'explorer ces différences.

J'estime toutefois que l'habileté à travailler à un niveau transnational, aussi bien qu'à un niveau transculturel, devrait être vue comme une compétence minimale chez un intervenant de groupe efficace. Cela ne veut pas dire que tous les intervenants doivent devenir des activistes sur le plan politique ou économique; cela signifie en revanche qu'ils doivent posséder l'assurance et la compétence, en tant qu'intervenants de groupe, pour franchir les frontières nationales et culturelles.

C'est de ça que nous avons besoin pour " problémiser ", pour reprendre Freire. Pas pour " faire des problèmes ", mais pour voir " comme un problème ". S'il n'y a pas de problème, il ne peut y avoir de solution. Mon hypothèse de base est que la capacité à travailler sur un plan bi-national est analogue à celle d'être bilingue. Les deux sont basées sur un processus de traduction. Cet aspect est fondamental, car trop souvent la rencontre internationale ou interculturelle est vue simplement comme une forme plus complexe $d u$ " $A$ rencontre $B$ en $X$ ». De manière schématique, nous pouvons représenter la rencontre transculturelle par :

\section{Culture $\mathrm{A} \rightarrow \longrightarrow \mathrm{X} \leftarrow \longleftarrow$ Culture $\mathrm{B}$}

En Europe, de nos jours, nous pouvons traverser les frontières sans passeport, sans contrôles douaniers, sans même changer de vitesse, de sorte que nous avons physiquement moins l'impression que nous allons nous trouver en présence d'une autre nation, d'une autre culture. Ainsi, ce modèle, en raison de sa simplicité, cache en fait un processus plus complexe qui révèle non seulement un, mais plusieurs points de rencontre culturels. J'ai adopté et développé un modèle tiré, comme pour le débat sur les emics et les etics, de la linguistique et de la traduction ${ }^{9}$.

9. P. Taylor et G. Pineau, «Pour une pédagogie de l'interculturel », dans C. Leray et E. Lorand, Dynamique interculturelle et autoformation, Paris, L'Harmattan, 1995. 


\section{Construction de la rencontre culturelle}

(1)

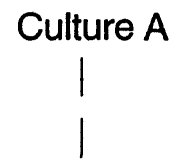

(2) Analyse

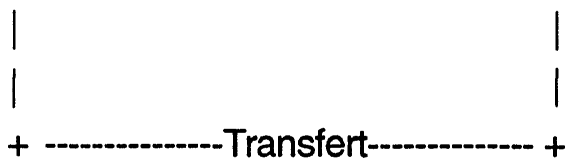

(3)
(5) Culture B<smiles>C1CCC1</smiles>

(4) Reconstruction

La réunion $\mathrm{A} \longleftrightarrow \longrightarrow \mathrm{B}$ constitue la partie visible que nous pouvons observer comme étant la pratique ou la performance. Ce qui demeure caché est l'infrastructure ou la métastructure de l'interculturalisme, les points 2-3-4. Toute véritable rencontre interculturelle doit être un mouvement réciproque de $A$ vers $B$ en passant par 2-3-4, et de $B$ vers $A$, en passant par ses propres 2-3-4 :

$$
A \rightarrow(2-3-4) \rightarrow B: B \rightarrow(2-3-4) \rightarrow A
$$

C'est dans ces zones de 2-3-4 que reposent les compétences de communication interculturelle.

En nous plaçant dans le cadre d'un modèle de développement de groupe ${ }^{10}$, intéressons-nous au stade de la discussion et de la dissension dans le groupe. On notera que dans les groupes avec une distance hiérarchique élevée les membres expriment les conflits et leur désaccord avec beaucoup de vigueur, des propos énergiques, des cris et même une attitude théâtrale. Par ailleurs, dans les groupes où la prise de risques se manifeste à un haut niveau, on minimise la colère évidente et la dissidence s'exprime non par une colère polie, retenue, mais par des remarques acerbes ou un silence insolent.

En situation de transculture, les deux groupes auraient d'abord la possibilité d'analyser le comportement, les valeurs et les stratégies qui leur sont propres. II leur faudrait ensuite transférer dans un

10. B. Tuckman, «Development Sequence in Small Groups », Psychological Bulletin, 1963, p. 384-399. 
registre différent leur compréhension de ce qu'ils ont à communiquer et reconstruire leur réponse normale de telle façon qu'elle soit significative pour l'autre. Ce qui n'est rien d'autre qu'un processus normal de traduction.

La traduction fait ressortir les différences aussi bien entre les langues qu'entre les cultures. L'analyse que nous en faisons nous aide à comprendre le caractère différent de l'autre, son " étrangeté " pour nous, et à voir jusqu'à quel point nous devons nous adapter pour trouver un terrain d'entente. II s'agit de l'übersertzen, ce qui signifie " traduire » et " faire traverser d'une rive à l'autre ".

Notre capacité de reconstruction dans une autre culture d'une façon qui soit significative dépend de notre aptitude à pénétrer cette culture de l'intérieur, à avoir une compréhension emic de ses valeurs, de ses comportements et de son organisation, ou tout au moins à être guidé par quelqu'un appartenant à cette culture. Si on nous indique, d'une façon ou d'une autre, qu' " on ne doit pas faire ça ici ", nous avons le choix : ou bien nous nous conformons à la culture hôte sans éprouver un sentiment de compromission; ou bien nous nous conformons, que nous éprouvions ou non un sentiment de compromission, parce que l'autre a le pouvoir de nous imposer le comportement qu'il veut nous voir adopter. Ou encore nous tentons de comprendre pourquoi un tel comportement serait considéré comme normal par eux et, en comparant le raisonnement qui le sous-tend avec notre propre système de normalité, nous pouvons nous en accommoder globalement, nous y adapter ou le refuser dans son ensemble. II nous faut accepter le fait que notre propre culture, qui nous semble tellement familière et naturelle, soit " étrange " pour d'autres, qui font une évaluation etic, et peut-être très juste, de ce que cette culture représente.

Une question essentielle est de savoir à quel point ce que nous tenons pour acquis, ce qui est censé être normal et courant, est véritablement explicité. Si nous supposons que " tout le monde sait ça " ou que " de toute évidence la meilleure chose à faire est de... ", il est hautement probable qu'une telle présomption échoue à reconnaître le niveau de différence apparent dans un échange international. Nous pouvons presque tracer un arc imaginaire qui révèle les contours de notre pratique. Plus les règles, la procédure, les normes et les valeurs sont explicitement reconnues dans le groupe, 
plus il y aura acceptation des différences inhérentes au groupe. Ou, à l'opposé, le niveau d'acceptation des différences dans le groupe sera directement rattaché au niveau d'expression et de clarification des suppositions culturelles et nationales.

Un groupe qui ne reconnaît pas les frontières culturelles et nationales court le risque d'être oppressif. L'affirmation voulant que nous soyons tous pareils et égaux est aussi dangereuse que celle qui confond différence et incompatibilité. II est sage de croire que, pour des relations personnelles aussi bien que pour des relations de groupe, nous devons trouver suffisamment de choses que nous avons en commun pour être capables de nous parler. Mais, en même temps, il nous faut trouver suffisamment de différences pour avoir quelque chose à dire.

Le mot, dit Bakhtin, est la moitié de l'Autre; il faut être deux pour dialoguer, tout comme il faut deux cultures-nations pour qu'il y ait échange transfrontalier. Nous pouvons nous arrêter à la frontière, ou nous pouvons la franchir. Mais nous devons d'abord savoir que cette frontière existe. " Être étranger » est une vue d'ici, vers là-bas. Mais de là-bas, nous paraissons aussi étrangers. Si, dans notre pratique de tous les jours, nous pouvons commencer à explorer en quoi consiste notre " étrangeté ", comment nous nous comportons, ce que nous considérons comme vrai et sensé, nous commencerons au moins à construire notre moitié de pont.

Intéressons-nous d'abord à la base. Examinons notre façon de gérer le pouvoir et l'individualité dans les groupes, d'être sensible au genre et de répondre à l'inconnu et au défi. Franchir les frontières culturelles et nationales peut ressembler à la marche vers la Terre promise. Mais comme Alinsky le disait si bien, « II y en a qui affirment : "J'y croirai quand je le verrai". Mais ce n'est pas vrai. Ils le verront plutôt quand ils y croiront. "

PaUL TAYLOR

Professeur Institut supérieur de formation permanente Université François-Rabelais 